\title{
The Gaussian Beam Summation and the Gaussian Launching Methods in Scattering Problem
}

\author{
Papa Ousmane Leye ${ }^{1}$, Ali Khenchaf', Philippe Pouliguen ${ }^{2}$ \\ ${ }^{1}$ Lab-STICC UMR CNRS 6285, ENSTA Bretagne, Brest, France \\ ${ }^{2}$ French General Directorate for Armament (DGA), Bagneux, France \\ Email: papa_ousmane.leye, Ali.Khenchaf@ensta-bretagne.fr, Philippe.Pouliguen@intradef.gouv.fr
}

How to cite this paper: Leye, P.O., Khenchaf, A. and Pouliguen, P. (2016) The Gaussian Beam Summation and the Gaussian Launching Methods in Scattering Problem. Journal of Electromagnetic Analysis and Applications, 8, 219-225.

http://dx.doi.org/10.4236/jemaa.2016.810020

Received: September 1, 2016

Accepted: October 7, 2016

Published: October 10, 2016

Copyright $\odot 2016$ by authors and Scientific Research Publishing Inc. This work is licensed under the Creative Commons Attribution International License (CC BY 4.0).

http://creativecommons.org/licenses/by/4.0/

\begin{abstract}
This paper is mainly devoted to application of the Gaussian beam summation technique in electromagnetic simulations problem. Gaussian beams are asymptotic solutions of the Helmholtz equation within the paraxial approximation. Since they are insensitive to ray transition region, several techniques based on Gaussian beam are used to evaluate high frequency EM wave equation, which overcome partially or fully the difficulties of singular regions (caustics, zero field in shadow zones). This paper concentrates on the explicit formulation of the electromagnetic field scattered from radar target. In this approach, when the incident field illuminates the target, the scattering is accounted in a complex weighing function. The wave field at a receiver is evaluated as superposition of Gaussian beams concentrated close to rays emerging from the target, passing through the neighbor of the receiver.
\end{abstract}

\section{Keywords}

Gaussian Beams, RCS, Scattering, Radar Targets

\section{Introduction}

Asymptotic methods using high-frequency approximations and the hypothesis of locally plane wave are based on the principle of rays. A direct application of these methods in a complex scenario (3D with objects of large sizes) is often confronted with problems of caustic, transition between shadow and highlighted region and requires a prohibitive calculation time due to the huge number rays necessary to describe the scene. In this paper, we test and apply contribution of two different techniques essentially based on Gaussian beam to estimate the RCS of canonical radar target which can be further applied to complex scheme. The principal raison of our interest in Gaussian beam for simulating the radiated field by use of Gaussian beams is that they eliminates 
several limitations of the ray method, in particular the difficulties connected with evaluating the wave field in singular regions (caustics, shadow regions, etc.). As compared to the classical asymptotic technique, there exists relatively little work in the open literature regarding application of Gaussian beam in diffraction problem.

The GBS technique was already formalized for diverse configurations [1] [2]. The approach proposed here is different. Nevertheless, our GBS formulation method is different. It consists of a representation of the wave field in the form of a single integral over elementary Gaussian beams concentrated in the vicinity of a single isolated ray which is referred to as the central or reference ray. The superposition of Gaussian beams as an asymptotic representation of a scalar wave field in the high frequency approximation was firstly suggested by V. M. Babich and T. F. Pankratova [3] in pure Mathematical studies. The consistent description of the Gaussian Beam method was given by Popov [4].

In the GBL formulation, the field radiated by the target illuminated by a Gaussian beam is decomposed into a plane wave spectrum. These Gaussian beams strike the surface from where they are reflected, and also diffracted by the reflector edge [5]. Finally the solution is obtained via an asymptotic evaluation of the radiation integral for the fields scattered, to within the physical optics approximation. This technique has attracted much attention, especially in the electromagnetic community.

In this paper, Section 2 presents general formulation of the two methods. In the GBS approach we derive general equations for the Gaussian beam at a point situated in the effective vicinity of the receiver and the resultant field. We develop numerical computation and investigate the sensitivity of the solution to the selection of certain of the Gaussian beam parameters which enable the required accuracy. For the GBL formulation, we give asymptotic evaluation of the radiation integral for the fields scattered from the target, to within the physical optics approximation. Section 3 presents formulation of the scattered field by a monostatic RCS of canonical target, which involves a single platform operating as transmitter and receiver, illuminated by horizontally polarized incident plane wave, using GBS and a rotationally symmetric Gaussian be amusing GBL. In the former, the normalization coefficient of the integral is identified by matching the analytical solution to the ray asymptotic solution as it happens to the Geometrical Optic (GO) solution. The two methods will be compared and simulation with canonical target will be compared to asymptotic methods (Physical Optic) and rigorous method (Method of Moment) to assess the advantages of these techniques.

\section{Formulation of the Method}

The Gaussian beam method, as a new approach for the computation of wave fields in high frequency approximation, was suggested by M.M. Popov [4] and V. Cerveny [6]. Consider an electromagnetic wave propagating in a homogeneous and isotropic medium which is being excited by a point source. In the GBS method, the final field in an observation point results from a fan of rays distributed in his vicinity. For each ray we derive a Gaussian beam propagating along the ray and then sum the contribution of 
each Gaussian beam to the receiver over all rays from the fan [7]. For any selected ray, we shall introduce a ray-centered coordinate system, $q_{1}, q_{2}$ and $s$ connected with it. A solitary Gaussian beam as a localized asymptotic solution to the Helmholtz Equation calculated at the receiver point, with local coordinates $s, q_{1}, q_{2}$ inside asymptotically small neighborhood of the central ray is given by [4].

$$
u\left(s, q_{1}, q_{2}, t\right)=\left(\frac{v}{\operatorname{det}[Q]}\right)^{\frac{1}{2}} \cdot \mathrm{e}^{-j \omega\left[t-\tau(s)-\frac{1}{2} \cdot\left(q^{\mathrm{T}} \cdot P \cdot Q^{-1} \cdot q\right)\right]}
$$

where $\tau(s)=\int_{s_{0}}^{s} \frac{\mathrm{d} s}{v}$ is the travel time from the source along the selected ray, $V$ is the propagation velocity, $q^{\mathrm{T}}$ represents the transpose of the vector $q$, the quantities $Q$ and $P$ are $2 \times 2$ matrix called "dynamic quantities" satisfying the system ODE (2) in variations, called the "dynamic ray tracing equations" (DRT) [8]. In homogeneous medium with wave speed $v$ supposed equal to the celerity denoted chere, the DRT can be written as

$$
\left\{\begin{array}{l}
\frac{\mathrm{d} Q}{\mathrm{~d} s}=c \cdot P \\
\frac{\mathrm{d} P}{\mathrm{~d} s}=0
\end{array}\right.
$$

To solve this above DRT system (2), the initial conditions must be specified at an arbitrary point $s=s_{0}$ on the central ray. We must solve this system of differential equations for $Q$ and $P$ with appropriate initial conditions. They must guarantee the following three conditions along the whole rays [9]:

- $\operatorname{det}[Q] \neq 0$.

- $P \times Q^{-1}$ is symmetric matrix, even though $P$ and $Q$ are not symmetrical.

- $\operatorname{Im}\left(P \times Q^{-1}\right)$ is a positive-definite matrix.

Here, we use Hill's [10] initial data for the Green's function. The initial values for $Q$ and $P$ are respectively.

$$
\left\{\begin{array}{l}
Q=\frac{\omega_{r} \cdot \omega_{0}^{2}}{c} \cdot I \\
P=\frac{j}{c} \cdot I
\end{array}, \quad S=s_{0} .\right.
$$

In (3), $\omega_{0}$ is the initial half beam width at the frequency $\omega_{r}$, $I$ is the identity matrix. An exhaustive discussion of the dependence of the Gaussian beam solution with beam with $\omega_{0}$ is given in [11] and [12]. The total amplitude at the receiver is given by the integral over all Gaussian beams characterized by their takeoff angle, denoted $\varphi$, from the source:

$$
u^{s f g}(M)=\int_{\delta} \Phi_{\varphi} \cdot u_{\varphi}\left(s, q_{1}, q_{2}\right) \cdot \mathrm{d} \delta
$$

In (4) the domain $\delta$ is centered on the central ray, it delimits the beams propagating in the neighbor of the central ray, chosen in such way that the Gaussian beam $u_{\varphi}\left(s, q_{1}, q_{2}\right)$ outside this domain do not contribute effectively to the wave field. $\delta$ is a 
cone with a vertex angle $\varphi$.

$$
\mathrm{d} \delta=\sin \varphi \cdot \mathrm{d} \varphi \cdot \mathrm{d} \vartheta, \quad \vartheta \in[0,2 \pi]
$$

In (4) $u_{\varphi}\left(s, q_{1}, q_{2}\right)$ is the Gaussian beam connected with the ray and $\Phi_{\varphi}$ is a quantity, generally complex-valued, which remains constant along the considered ray but may differ from ray to ray. It is called complex weight function.

The above solution is influenced by parameters which specify the properties of Gaussian beams used, mainly their width. We must learn how to select the parameters of computation to obtain the results with the required accuracy. We analyze the behavior of GBS solution for different beam width and investigate how this proposed solution offer better result near singularities where classical ray methods fail. We use simplest ray theory approximation for comparison, considering the direct ray. The results of the first numerical experiments were presented in [11] and [13]. We compare the GBS and the ray theory approximation considering a point source and a receiver on the central ray.

For homogeneous medium, denoting $r$ the distance to an observation point $M$, the ray asymptotic solution of the Helmholtz Equation is given by

$$
u(M)=\frac{\mathrm{e}^{j \cdot \frac{\omega}{c} \cdot r}}{4 \pi r}
$$

On the other hand, the GBS integral, in Equation (4), may be evaluated asymptotically using the saddle-point method. Thus, this result must coincides with the above ray asymptotic solution in regular region. Matching both asymptotic solution of (4) and (6) we can determine the complex weight function $\Phi_{\varphi}$. Integral (4) is evaluated by numerical quadrature with regular increment $\Delta \varphi$

$$
u(M)=2 \cdot \pi \cdot \sum_{k=1}^{N} \Phi_{\varphi_{k}} \cdot u_{\varphi_{k}} \cdot \sin \left(\varphi_{k}\right) \cdot \Delta \varphi_{k}
$$

Figure 1 left compares, field amplitude calculated by GBS method and ray asymptotic solution of the Helmholtz equation for $\omega_{0}=5 \lambda, 8 \lambda, 12 \lambda$ and 15,$N=400 . r$ is the distance from the source to the receiver. Red, green, blue and magenta line correspond to the GBS solution for different beam width, respectively $5 \lambda, 8 \lambda, 12 \lambda$ and $15 \lambda$. The beam initial width must be chosen optimally to guarantee sufficient accuracy. The role of $\omega_{0}$ has been investigated by several authors, see [14] [15]. The relative error between GBS solution and ray asymptotic solution shown in Figure 1 right is normalized by the asymptotic solution. We observe that for $\omega_{0}=15 \lambda$ the relative error remains below $4 \%$ even at $10 \mathrm{~km}$ from the source and the GBS solution match the ray asymptotic solution. In addition, one should note that the GBS method exhibit no singularities when passing by the source point $(r=0)$, unlike the ray asymptotic solution. The proof of this result relies on the theory of systems of linear first order differential equations [16].

The above GBS formulation will now be compared to the alternative approach called GBL. This technique consists in calculating the radiation integral of the fields scattered by the target illuminated by a Gaussian beam, within the physical optics approximation. 

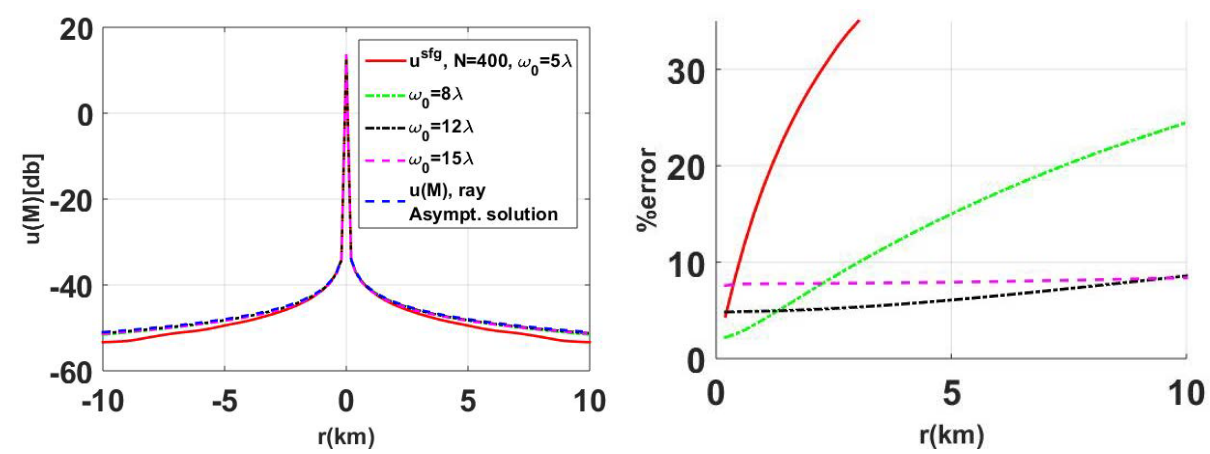

Figure 1. RCS of a flat plate, illuminated by a vertically polarized incident wave, computed using GBL method, GBS, and PO, as a function of transmitter elevation angle in monostatic configuration for beam width $\omega_{0}=2 \lambda$.

For the incident Gaussian beam, the incident magnetic field can be written as [5]

$$
\boldsymbol{H}_{i}\left(\boldsymbol{r}_{\boldsymbol{i}}\right)=\boldsymbol{H}_{i}(0) \sqrt{\frac{\rho_{i}+j b_{i}}{z_{i}+\rho_{i}+j b_{i}}} \cdot \mathrm{e}^{-j k\left(\frac{\left.z_{i}+\frac{x_{i}^{2}+y_{i}^{2}}{z_{i}+\rho_{i}+j b_{i}}\right)}{z^{2}} .\right.}
$$

The PO integral, integration of the incident Gaussian beam on the reflector surface, give the electric fields scattered from the surface. The integral can be written as

$$
\boldsymbol{E}(r)=\frac{j k}{4 \pi} \iint_{\Sigma}\left[\frac{Z_{0}}{\sqrt{\varepsilon_{r}}} \cdot \boldsymbol{R} \times \boldsymbol{R} \times\left(\boldsymbol{e}_{z} \times \boldsymbol{H}_{i}\left(\boldsymbol{r}_{i}\right)\right)\right] \cdot \frac{\mathrm{e}^{-j k R}}{R} \cdot \mathrm{d} s
$$

where $\boldsymbol{r}_{i}$ is the position vector in the incident Gaussian beam, $\rho_{i}$ is the distance from the waist of the incident Gaussian beam to point $\mathrm{O}$ on $\Sigma$ (see Figure 2 left) and. $b=\frac{k \omega_{0}^{2}}{2}$, where $\omega_{0}$ is the half beam width.

\section{Numerical Computation}

We introduce the principle of calculation of the scattered field by a flat plate, illuminated by a plane wave, using GBS and rotationally symmetric Gaussian beams propagating in the $Z_{i}$ direction using GBL, at $10 \mathrm{GHz}$. As described in Section 2, GBS is applied at the receiver and the weight function is identified by comparing the solution of (4), using steepest decent method, to those of Geometrical Optic.

Using equation (7), we have computed the scattered field applying GBS. Using equation (8) in (9) and solving the integral, we have computed the scattered field applying GBL formulation as in [17]. In Figure 2, we shows monostatic scattering from a rectangular flat plate of sides $1 \mathrm{~m} \times 1 \mathrm{~m}$ which lies in the $(x, y)$ plane (Figure 2 left). We set the azimuth angles $\varphi_{i}$ to zero and we show comparison between GBS, GBL, for a beam width $\omega_{0}=2 \lambda$, PO and Mom simulated using FEKO. The transmitter elevation angle varies from $\theta_{i}=-90^{\circ}$ to $\theta_{i}=90^{\circ}$. The GBS result (blue line) and GBL technique (bashed red line) match rigorously the PO solution (black line) and Mom (light blue line) for the main beams. One can see that GBS and GBL technique are superposed to the PO solution. PO and Mom accurately models the main beam, and the modeled 

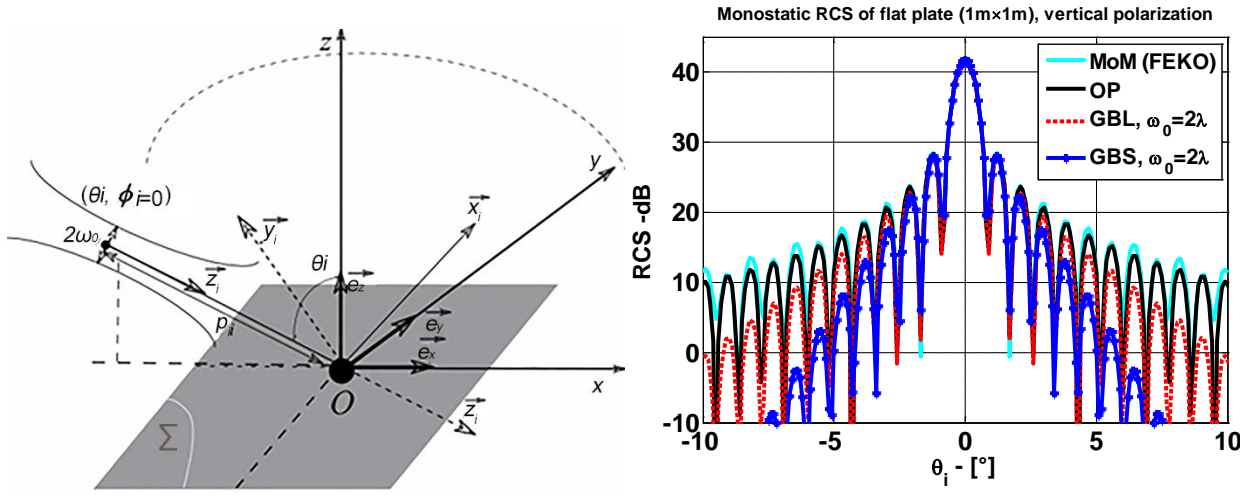

Figure 2. RCS of a flat plate, illuminated by a vertically polarized incident wave, computed using GBL method, GBS, and PO, as a function of transmitter elevation angle in monostatic configuration for beam width $\omega_{0}=2 \lambda$.

peak width and location match GBS and GBL technique. GBS and GBL accurately model the main beam, without mitigation, the diagrams are also consistent, and the modeled peak width and location match the PO solution. Comparing GBS and GBL one can observe a progressive degradation on GBL solution into the deep shade according to the mitigation. It is questionable in general whether the accuracy gained by using GBS would justify the additional complexity of the solution method.

\section{Conclusion}

The main purpose of the asymptotic approximation technique in electromagnetism is to give effective numerical solution of the wave equation combining accuracy and better computing time. We treat two different approaches to compute the electromagnetic field radiated from radar target. The beam methods promise several advantages over ray methods, particularly on caustic problem. In the GBS formulation, the total field at the receiver is represented by the integral over all Gaussian beams propagating in the neighbor of the receiver. Established solution is compared to another approach also based on Gaussian beam which consists of calculating the field radiated by the object illuminated by a Gaussian beam by decomposing it into a plane wave spectrum. The final field is the contribution of beams interacting with the target. This comparison shows that GBS and GBL give high accurate representation of the scattered field. Simulation with canonical target is shown and will be extended to complex target.

\section{Acknowledgements}

The work presented in this paper is supported by DGA (Direction Générale de l'Armement, France)-MRIS in the frame of SOFAGEMM project.

\section{References}

[1] Katsav, M. and Heyman, E. (2009) Gaussian Beam Summation Analysis of Half Plane Diffraction: A Full 3D. Formulation, 313-317.

[2] George, T. and Pouliguen, P. (2013) Sommations de Faisceaux Gaussiens appliquée aux 
modèles électromagnétiques 3D. Journée Thématique DGA: Méthodes Hautes Fréquences pour les calculs de signatures radar et d'antennessurporteurs, Paris.

[3] Babich, V.M. and Pankratova, T.F. (1973) On Discontinuities of the Green Function of Mixed Problem for Wave Equation with Variable Coefficients. In: Problem of Mathematical Physics, Vol. 6, Leningrad University Press, 9-27.

[4] Popov, M.M. (1982) A New Method of Computation of Wave Fields Using Gaussian Beams. Wave Motion, 4, 85-97. http://dx.doi.org/10.1016/0165-2125(82)90016-6

[5] Chou, H.T. and Pathak, P.H. (1997) Uniform Asymptotic Solution for Electromagnetic Reflection and Diffraction of an Arbitrary Gaussian Beam by a Smooth Surface with an Edge. Radio Science, 32, 1319-1336. http://dx.doi.org/10.1029/97RS00713

[6] Cerveny, V. (1982) Expansion of a Plane Wave into Gaussian Beams. Studia Geophysica et Geodaetica, 26, 120-131. http://dx.doi.org/10.1007/BF01582305

[7] Cerveny, V. (2001) Seismic Ray Theory. Cambridge University Press, Cambridge. http://dx.doi.org/10.1017/CBO9780511529399

[8] Popov, M.M. (1981) A New Method of Computation of Wave Fields in High Frequency Approximation. In: Mathematical Problems of Theory of Propagation of Waves, Vol. 11, Nauka, Leningrad, 195-216.

[9] Cerveny, V. (1985) Gaussian Beam Synthetic Seismograms. Journal of Geophysics, 58, 4472.

[10] Hill, N.R. (1990) Gaussian Beam Migration. Geophysics, 55, 1416-1428. http://dx.doi.org/10.1190/1.1442788

[11] Cerveny, V. (1982) Computation of Wave Field in Homogeneous Media. Geophysical Journal of the Royal Astronomical Society, 70, 109-128. http://dx.doi.org/10.1111/j.1365-246X.1982.tb06394.x

[12] White, B.S., Norris, A., Bayliss, A. and Burridge, R. (1987) Some Remarks on the Gaussian Beam Summation Method. Geophysical Journal of the Royal Astronomical Society, 89, 579636. http://dx.doi.org/10.1111/j.1365-246X.1987.tb05184.x

[13] Kachalov, A.P. and Popov, M.M. (1981) Application of the Gaussian Beam Method to Computations of High Frequency Wave Fields. Geophysical Journal International, 81, 205214. http://dx.doi.org/10.1111/j.1365-246X.1985.tb01359.x

[14] Stephen, R.A. (1999) Optimum and Standard Beam Widths for Numerical Modeling of Interface Scattering Problems. Journal of the Acoustical Society of America, 107, 1095-1102. http://dx.doi.org/10.1121/1.428399

[15] Madariaga, R. (1984) Gaussian Beam Synthetic Seismograms in a Vertically Varying Medium. Geophysical Journal of the Royal Astronomical Society, 79, 589-612. http://dx.doi.org/10.1111/j.1365-246X.1984.tb02243.x

[16] Bleistein, B. (2008) Mathematics of Modeling, Migration and Inversion with Gaussian Beams. Colorado, USA.

[17] Chou, H.T., Pathak, P. and Burkholder, R.J. (2001) Novel Gaussian Beam Method for the Rapid Analysis of Large Reflector Antennas. IEEE Transactions on Antennas and Propagation, 49, 880-893. http://dx.doi.org/10.1109/8.931145 
Submit or recommend next manuscript to SCIRP and we will provide best service for you:

Accepting pre-submission inquiries through Email, Facebook, LinkedIn, Twitter, etc. A wide selection of journals (inclusive of 9 subjects, more than 200 journals)

Providing 24-hour high-quality service

User-friendly online submission system

Fair and swift peer-review system

Efficient typesetting and proofreading procedure

Display of the result of downloads and visits, as well as the number of cited articles

Maximum dissemination of your research work

Submit your manuscript at: http://papersubmission.scirp.org/

Or contact jemaa@scirp.org 